\title{
Adamantinomatous craniopharyngioma associated with a compromised blood-brain barrier: patient series
}

\author{
*Eric W. Prince, BS, ${ }^{1}$ Lindsey M. Hoffman, DO, ${ }^{5}$ Trinka Vijmasi, MD, ${ }^{1}$ Kathleen Dorris, MD, ${ }^{2,6}$ Jennifer A. McWilliams, PhD, ${ }^{3}$ \\ Kimberly R. Jordan, PhD, ${ }^{3}$ David M. Mirsky, MD, ${ }^{4}$ and Todd C. Hankinson, MD ${ }^{1,2,6}$ \\ Departments of ${ }^{1}$ Neurosurgery, ${ }^{2}$ Pediatrics, ${ }^{3}$ Immunology and Microbiology, and ${ }^{4}$ Radiology, University of Colorado School of Medicine and Children's Hospital Colorado, \\ Aurora, Colorado; ${ }^{5}$ Division of Hematology/Oncology, Phoenix Children's Hospital, Phoenix, Arizona; and ${ }^{6}$ Morgan Adams Foundation Pediatric Brain Tumor Research Program, \\ Denver, Colorado
}

\begin{abstract}
BACKGROUND Adamantinomatous craniopharyngioma (ACP) is a highly morbid adult and pediatric brain tumor derived from epithelial remnants of the craniopharyngeal canal (Rathke's pouch), which gives rise to the anterior pituitary gland. Standard therapy includes maximal safe resection with or without radiation therapy. Systemic antitumor therapy remains elusive. Immune-related paracrine signaling involving the interleukin-6 receptor (IL-6R) may contribute to ACP pathogenesis. Tocilizumab, a recombinant humanized monoclonal antibody against IL-6R, is approved by the US Food and Drug Administration but does not cross an intact blood-brain barrier.

OBSERVATIONS In a phase 0 trial design, a single dose of tocilizumab was delivered intravenously before clinically indicated surgical intervention in 3 children with ACP. The presence of tocilizumab was assayed in plasma, tumor tissue, tumor cyst fluid, and cerebrospinal fluid $(n=1)$ using a novel enzyme-linked immunosorbent assay. Tocilizumab reached ACP tumor tissue and/or cyst fluid after one systemic dose in every patient.
\end{abstract}

LESSONS This finding helps explain extant data that indicate tocilizumab may contribute to ACP therapy. It further indicates that ACP does not reside behind an intact blood-brain barrier, dramatically broadening the range of potential antitumor therapies against this tumor. This has substantial implications for the design of future clinical trials for novel therapies against ACP in both children and adults.

https://thejns.org/doi/abs/10.3171/CASE2150

KEYWORDS adamantinomatous craniopharyngioma; craniopharyngioma; tocilizumab; blood-brain barrier; monoclonal antibody

Adamantinomatous craniopharyngioma (ACP) is a World Health Organization grade I tumor derived from epithelial remnants of the primordial craniopharyngeal canal (Rathke's pouch), which gives rise to the anterior pituitary gland. ACP exhibits a bimodal age distribution, with peak incidences at 5-15 and 45-60 years. ${ }^{1,2}$ In contrast to its benign histological appearance, the manifestations of ACP are often severe and debilitating, stemming from proximity to and effect on the optic pathway, pituitary gland, and hypothalamus. ACP has been associated with the lowest quality-of-life scores of any pediatric brain tumor. ${ }^{3}$

As opposed to the situation for papillary craniopharyngioma ${ }^{4}$ and partially due to an incomplete understanding of the pathobi- ology of ACP, no systemic chemotherapy has been established as a viable option. Immune-related paracrine signaling may help drive ACP pathogenesis. Among the potential contributors to this process are interleukin (IL)- 6 and IL-6 receptor (IL-6R), which are found in high concentrations in ACP cyst fluid and tumor tissue, respectively. ${ }^{5-8}$ Tocilizumab, a recombinant humanized monoclonal antibody against soluble and membrane-bound IL$6 \mathrm{R}$, is approved by the US Food and Drug Administration for juvenile idiopathic arthritis or chimeric antigen receptor $T$ cell-induced cytokine release syndrome in children older than 2 years of age. We demonstrated therapeutic potential for tocilizumab against $A C P$ in a recent report describing 2 patients with refrac-

ABBREVIATIONS ACP = adamantinomatous craniopharyngioma; $\mathrm{BBB}=$ blood-brain barrier; $\mathrm{C}_{\max }=$ maximum concentration; CNS $=$ central nervous system; CSF = cerebrospinal fluid; ELISA = enzyme-linked immunosorbent assay; IL-6 = interleukin-6; IL-6R = interleukin-6 receptor. INCLUDE WHEN CITING Published May 10, 2021; DOI: 10.3171/CASE2150. SUBMITTED January 23, 2021. ACCEPTED February 2, 2021.

*E.W.P. and L.M.H. contributed equally to this work.

(C) 2021 The authors, CC BY-NC-ND 4.0 (http://creativecommons.org/licenses/by-nc-nd/4.0/). 


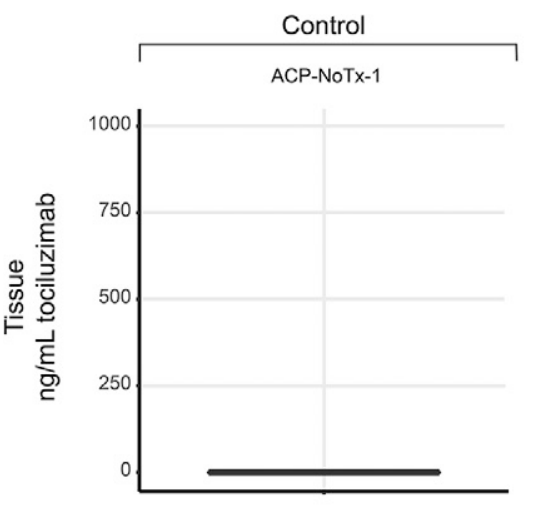

ACP-NoTX-2
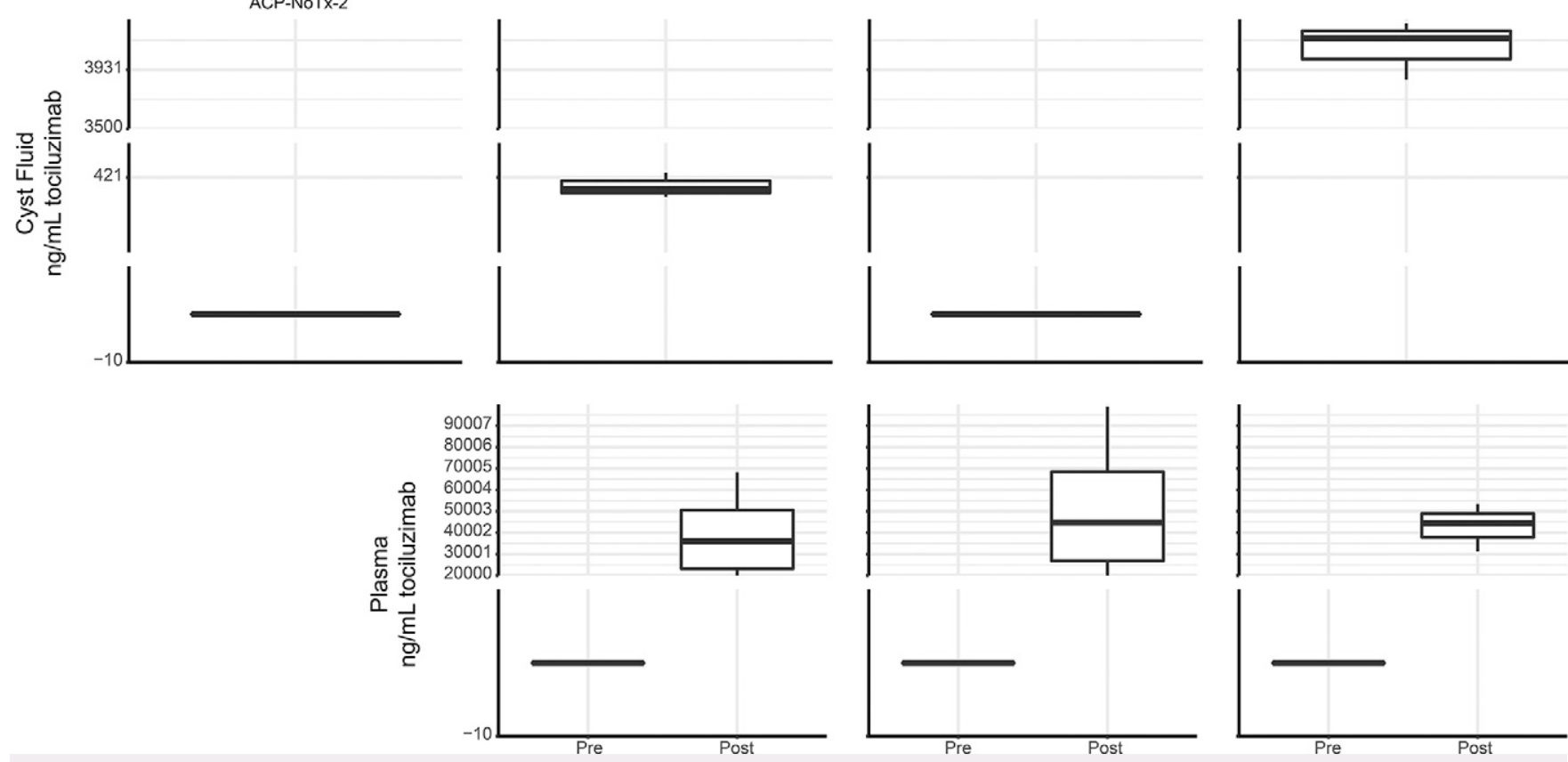

FIG. 1. Mean tocilizumab concentration measured in ACP tumor tissue (top), tumor cyst fluid (middle), and plasma (bottom). ACP-1 and ACP-2 received tocilizumab 4-6 hours before tissue sampling. ACP-3 received tocilizumab 25 hours before tissue sampling. The boxes represent the 25th to 75th centiles (the interquartile range $[I Q R])$. The mean is shown as the horizontal line in the box. The vertical lines that extend are $1.5 \mathrm{IQR}$ and $0.5 \mathrm{IQR}$. Tx $=$ treatment.

tory ACP who exhibited response to tocilizumab with or without bevacizumab. ${ }^{9}$

The microvasculature of the central nervous system (CNS), collectively termed the "blood-brain barrier" (BBB), tightly controls the passage of ions, molecules, and cells into the CNS to maintain homeostasis within this evolutionarily protected space. Drug penetration across the BBB is impacted by factors such as molecular size, lipid solubility, and efflux transporters. ${ }^{10}$ Regarding molecular size, most publications define an absolute cutoff of 400-600 Da, ${ }^{11}$ and the largest substance reported to cross the BBB to date is 7.8 $\mathrm{kDa} .{ }^{12}$ Though the BBB is believed to be disrupted in some CNS tumors, many potential therapeutic agents are found at inadequate concentrations in cerebrospinal fluid (CSF) and brain tumor tissue, likely secondary to nonuniform BBB disruption and/or factors such as drug efflux.

Though formal human BBB penetration studies for tocilizumab have not been reported, its large molecular weight $\left(148 \mathrm{kDa}^{13}\right)$ implies that it should not traverse the BBB. This supposition was supported by
Nellan et al., ${ }^{14}$ who evaluated the pharmacokinetics and CSF concentration of tocilizumab after intravenous, intrathecal, and intranasal delivery in nonhuman primates. The authors found that only intraventricular delivery achieved therapeutic CSF concentrations. After intravenous administration, the maximum concentration $\left(C_{\max }\right)$ in the CSF was 0.097 $\mu \mathrm{g} / \mathrm{mL}$ at $24-72$ hours after drug administration. After a single intravenous dose of tocilizumab, plasma levels increased at 2-8 hours, but CSF levels did not begin to rise until after 8 hours, peaking at 24-48 hours. The CSF/plasma ratio of the $C_{\max }$ of tocilizumab was $0.415 \times$ $10^{-3}$.

We hypothesized that ACP is associated with impaired BBB protection. This may result from the tumor's origin from precursors of the anterior pituitary gland, which functionally resides outside the $\mathrm{BBB}$, presumably to facilitate secretion of hormones into the systemic circulation. The clinical relevance of this hypothesis relates to maximizing the pool of potential antitumor agents for investigation against ACP. Evidence that ACP does not dwell behind an intact BBB can facilitate the progress of agents that show promise in the 


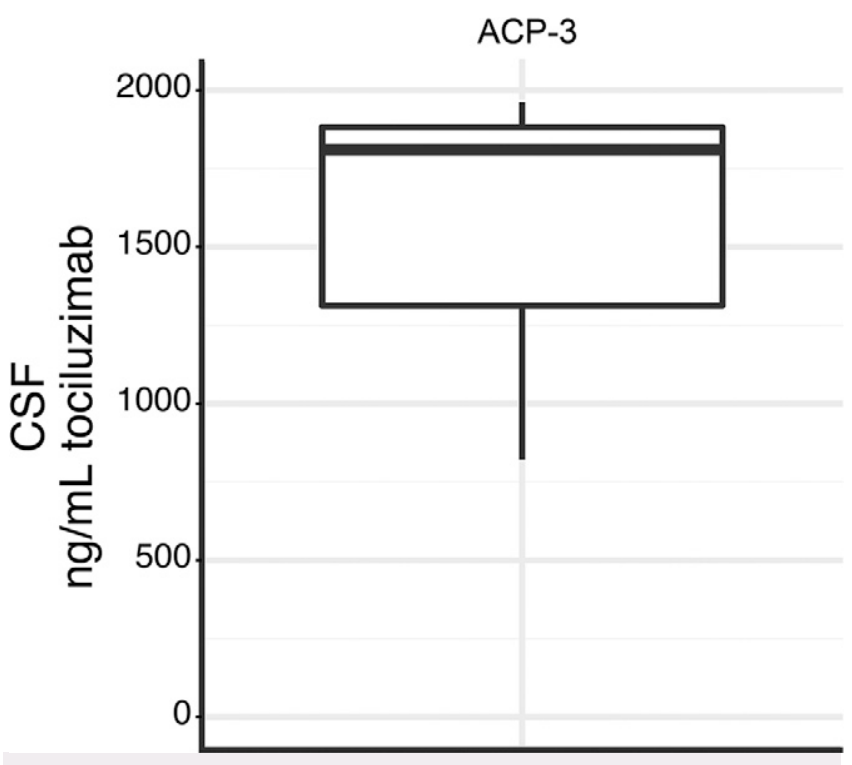

FIG. 2. Tocilizumab concentration measured in CSF of patient ACP-3, who received tocilizumab 25 hours before tissue sampling.

preclinical setting toward clinical trials. Large molecules, including monoclonal antibodies such as tocilizumab, will not be unnecessarily excluded from further investigation due to concerns for failure to cross the BBB. Such evidence could significantly broaden the pool of potential antitumor treatments for patients with ACP.

\section{Study Description}

Three children were enrolled in a phase 0 clinical trial (NCT03970226). After parents/caregivers provided informed consent, each patient received a single dose of intravenous tocilizumab infused in normal saline through a dedicated intravenous line before scheduled craniotomy for ACP resection. Two patients (ACP-1 and ACP-2) received the infusion 4-6 hours before tissue sampling, while the third (ACP-3) received the infusion 25 hours before tissue sampling. Free and bound tocilizumab were measured using an enzyme-linked immunosorbent assay (ELISA). ${ }^{13}$ Peripheral blood was drawn within 15 minutes before study drug administration and at the time of tumor tissue biopsy.

Tocilizumab was detected in ACP tumor tissue, cyst fluid, or CSF in each patient and in both tumor and cyst fluid in 2 of 3 patients (Fig. 1). CSF was sampled in only 1 patient (ACP-3), and tocilizumab was detected. The $\mathrm{CSF} \mathrm{C}_{\max }$ /plasma $\mathrm{C}_{\max }$ ratio for tocilizumab in this patient was 0.172 (Fig. 2).

\section{Discussion}

\section{Observations}

The identification of tocilizumab at detectable levels in CSF, cyst fluid, and tumor tissue after a single dose supports the proposition that systemically administered biologically promising therapeutic agents, such as tocilizumab or other large molecules, merits careful investigation in clinical trials for patients with ACP. While CSF was sampled in only a single patient, the CSF $C_{\max }$ /plasma $\mathrm{C}_{\max }$ ratio for tocilizumab was approximately 400 times higher than that identified by Nellan et al. in nonhuman primates with an intact BBB. ${ }^{14}$ It is notable, however, that the tocilizumab levels detected varied by both compartment and patient. Furthermore, tocilizumab saturates the IL-6R at a concentration of $0.1 \mu \mathrm{g} / \mathrm{mL}$, with complete inhibition at $4 \mu \mathrm{g} / \mathrm{mL}^{15}$ These levels were variably achieved in the specimens assayed in this study. These data, when combined with the clinical responses to tocilizumab that we previously reported, ${ }^{9}$ bolster the supposition that repeat dosing may produce higher effective drug concentrations. In addition, recent gene expression data indicate that therapeutic targets identified in pediatric ACP may also have relevance in adult $A C P{ }^{16}$ Thus, further investigations with larger patient numbers and additional agents are merited.

\section{Lessons}

This small phase 0 study demonstrates compelling evidence that the humanized monoclonal antibody tocilizumab effectively reaches ACP tumor and cyst fluid after systemic delivery. This finding supports existing data that indicate tocilizumab may have a role in systemic therapy against ACP. Furthermore, these findings imply that the BBB associated with ACP is compromised relative to other regions of the CNS. This has substantial implications for the design of clinical trials for novel therapies against ACP in both children and adults because agents that might have otherwise been discarded due to poor BBB penetration may now merit consideration. Initial clinical trials leveraging this information are underway.

\section{Acknowledgments}

We thank our patients for providing tissue specimens and receiving medication infusion. We thank the Human Immune Monitoring Shared Resource within the University of Colorado Human Immunology and Immunotherapy Initiative for their expert assistance in analysis of ELISA results. This work was supported by the Brain Tumour Charity (GN-000522; T.C.H.) and the National Cancer Institute (R03 CA235200; T.C.H.). Shared resources were supported through the University of Colorado Cancer Center Support Grant (P30CA046934; T.C.H., T.V., D.M.M., E.W.P., L.M.H., K.D., J.A.M., K.J.).

\section{References}

1. Müller HL, Merchant TE, Warmuth-Metz M, et al. Craniopharyngioma. Nat Rev Dis Primers. 2019;5(1):75.

2. Hankinson TC, Fields EC, Torok MR, et al. Limited utility despite accuracy of the national SEER dataset for the study of craniopharyngioma. J Neurooncol. 2012;110(2):271-278.

3. Foreman NK, Faestel PM, Pearson J, et al. Health status in 52 long-term survivors of pediatric brain tumors. J Neurooncol. 1999; 41(1):47-53.

4. Brastianos PK, Shankar GM, Gill CM, et al. Dramatic response of BRAF V600E mutant papillary craniopharyngioma to targeted therapy. J Natl Cancer Inst. 2015;108(2):djv310.

5. Apps JR, Carreno G, Gonzalez-Meljem JM, et al. Tumour compartment transcriptomics demonstrates the activation of inflammatory and odontogenic programmes in human adamantinomatous craniopharyngioma and identifies the MAPK/ERK pathway as a novel therapeutic target. Acta Neuropathol. 2018;135(5):757-777.

6. Donson AM, Apps J, Griesinger AM, et al. Molecular analyses reveal inflammatory mediators in the solid component and cyst fluid of human adamantinomatous craniopharyngioma. J Neuropathol Exp Neurol. 2017;76(9):779-788. 
7. Gump JM, Donson AM, Birks DK, et al. Identification of targets for rational pharmacological therapy in childhood craniopharyngioma. Acta Neuropathol Commun. 2015;3:30.

8. Zhou J, Zhang C, Pan J, et al. Interleukin-6 induces an epithelialmesenchymal transition phenotype in human adamantinomatous craniopharyngioma cells and promotes tumor cell migration. Mol Med Rep. 2017;15(6):4123-4131.

9. Grob S, Mirsky DM, Donson AM, et al. Targeting IL-6 is a potential treatment for primary cystic craniopharyngioma. Front Oncol. 2019;9:791.

10. Daneman R, Prat A. The blood-brain barrier. Cold Spring Harb Perspect Biol. 2015;7(1):a020412.

11. Banks WA. Characteristics of compounds that cross the blood-brain barrier. BMC Neurol. 2009;9(suppl 1):S3.

12. Pan W, Kastin AJ. Changing the chemokine gradient: CINC1 crosses the blood-brain barrier. J Neuroimmunol. 2001;115(1-2): 64-70.

13. Highlights of Prescribing Information. Actemra. Accessed 7 April 2021. https://www.gene.com/download/pdf/actemra_prescribing.pdf.

14. Nellan A, McCully CML, Cruz Garcia R, et al. Improved CNS exposure to tocilizumab after cerebrospinal fluid compared to intravenous administration in rhesus macaques. Blood. 2018;132 (6):662-666.

15. Shetty A, Hanson R, Korsten $P$, et al. Tocilizumab in the treatment of rheumatoid arthritis and beyond. Drug Des Devel Ther. 2014;8: 349-364.

16. Prince $E$, Whelan R, Donson A, et al. Transcriptional analyses of adult and pediatric adamantinomatous craniopharyngioma reveals similar expression signatures regarding potential therapeutic targets. Acta Neuropathol Commun. 2020;8(1):68.

\section{Disclosures}

Dr. Hoffman reports personal fees from Astra Zeneca outside the submitted work.

\section{Author Contributions}

Conception and design: Hankinson, Hoffman, Dorris, Mirsky. Acquisition of data: Hankinson, Prince, Hoffman, Vijmasi, McWilliams, Jordan. Analysis and interpretation of data: Hankinson, Prince, Hoffman, McWilliams, Jordan. Drafting the article: Hankinson, Hoffman. Critically revising the article: Hankinson, Hoffman, Dorris, Mirsky. Reviewed submitted version of manuscript: Hankinson, Prince, Hoffman, Jordan, Mirsky. Approved the final version of the manuscript on behalf of all authors: Hankinson. Statistical analysis: Prince. Administrative/technical/ material support: Prince, McWilliams. Study supervision: Hankinson, Dorris.

\section{Supplemental Information}

Previous Presentations

Portions of this work were presented in digital poster form at the 49th Annual Meeting of American Association of Neurological Surgeons/ Congress of Neurological Surgeons Joint Section on Pediatric Neurosurgery virtual meeting held on December 2-4, 2020, and at the 19th International Symposium on Pediatric Neuro-Oncology, Karuizawa, Nagano, Japan, held on December 13-16, 2020.

\section{Correspondence}

Todd C. Hankinson: Children's Hospital Colorado, University of Colorado School of Medicine, Aurora, CO. todd.hankinson@childrenscolorado.org. 